\title{
G

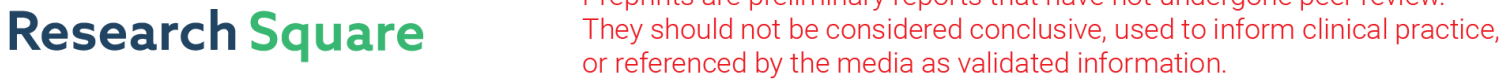 \\ Identification of a Gene Set Correlated with Immune Status in Ovarian Cancer by Transcriptome-Wide Data Mining
}

\section{Lili Fan}

Department of Pathology, Xiangya Hospital, School of Basic Medical Sciences, Central South University, Changsha, Hunan Province, China

Han Lei

Department of Pathology, Xiangya Hospital, School of Basic Medical Sciences, Central South University, Changsha, Hunan Province, China

\section{Ying Lin}

Department of Immunology, School of Basic Medical Sciences, Central South University, Changsha, Hunan Province, China

\section{Tianxiang Zhang}

Department of Immunobiology, Yale University School of Medicine, New Haven, CT, USA

\section{Zhengwei Zhou}

Department of Pathology, Xiangya Hospital, School of Basic Medical Sciences, Central South University, Changsha, Hunan Province, China

\section{Guang Shu}

School of Basic Medical Sciences, Central South University, Changsha, Hunan Province, China

\section{Zhipeng Yan}

Hunan Cancer Hospital/the affiliated Cancer Hospital of Xiangya School of Medicine, Central South University, Changsha, Hunan Province, China

\section{Haotian Chen}

Department of Pathology, Xiangya Hospital, School of Basic Medical Sciences, Central South University, Changsha, Hunan Province, China

Gang Yin ( $\nabla$ gangyin@csu.edu.cn )

Central South University Xiangya School of Medicine

\section{Research}

Keywords: ovarian cancer, TMB, immune cells infiltration, survival prognosis

Posted Date: September 15th, 2020

DOl: https://doi.org/10.21203/rs.3.rs-49922/v1 
License: (c) (i) This work is licensed under a Creative Commons Attribution 4.0 International License. Read Full License 


\section{Abstract}

Background: Immune checkpoint blocking (ICB) immunotherapy has achieved great success in the treatment of various malignancies. Although not have been approved for the treatment of ovarian cancer (OC), it has been actively tested for the treatment of OC. Response to immunotherapy closely relies on the immune profile of tumors. However, biomarkers that could indicate the immune status of $\mathrm{OC}$ and predict the response to ICB are rare.

Method: We downloaded RNAseq and clinical data of ovarian cancer from The Cancer Genome Atlas (TCGA). Up-regulated differentially expressed genes (Up-DEGs) were identified by analyzing the gene expression levels using the "limma, version=3.8" package in R software. Gene ontology (GO) and Kyoto Encyclopedia of Genes and Genomes (KEGG) pathway enrichment analyses were performed in the "GSVA" and "limma" package in R software. Furthermore, gene set enrichment analysis (GSEA) and investigation of protein-protein interaction (PPI) networks were performed to clarify the function of UpDEGs in both $\mathrm{TMB}^{\text {high }}$ group and immunity ${ }^{\text {high }}$ group. The correlation of genes with overall survival was also analyzed by conducted Kaplan-Meier survival analysis. Finally, TIMER and TIDE were used to predict gene immune function and its association with immunotherapy.

Results: Data analysis revealed both $\mathrm{TMB}^{\text {high }}$ and immunity ${ }^{\text {high }}$ were significantly related to better survival of OC. Four genes, CXCL13, FCRLA, MS4A1, and PLA2G2D were found positively correlated with better prognosis of $\mathrm{OC}$ and mainly involved in immune response-related pathways. We also found that these four genes were positively correlated with better response to immune checkpoint blockade-based immunotherapy according to available data of a melanoma cohort.

Conclusion: CXCL13, FCRLA, MS4A1, and PLA2G2D may be used as potential therapeutic biomarkers for reflecting $\mathrm{OC}$ immune status and predicting response to immunotherapy.

\section{Introduction}

Ovarian cancer (OC) is the most fatal gynecological tumor around the world. Due to the lack of specific symptoms and effective screening strategies[1], more than $70 \%$ of patients are in the middle or late stages at the time of diagnosis resulting in an increased risk of recurrence and poor prognosis[2]. Despite having various treatments, the five-year survival rate of late-stage patients was no more than $35 \%[3]$. Up to $70 \%$ of patients who were treated with traditional therapies, including chemotherapy and targeted therapy, relapse after 12 to 18 months[4], highlighting the importance of exploring novel therapies and identify a new prognostic and predictive biomarker for treatment of OC.

As a rising novel treatment, PD-1/PD-L1 blockade based immunotherapy has demonstrated promising efficacy in treating various types of solid tumors[5]. Yet current clinical trials on OC have shown that this immunotherapy can only benefit a small part of the patients while a large number of patients have either limited or no response[6, 7]. Thus, it's essential to choose the right patients for treatment. Ample of clinical data indicate that pre-existing favorable tumor immune microenvironment (TIME) are 
prerequisites for PD-1/PD-L1 blocking antibody-mediated immunotherapy[8, 9]. However, clinical biomarkers the can predict the immune status of $\mathrm{OC}$ are rare.

Tumor mutation burden (TMB) has been demonstrated as a potential biomarker to predict responses to immunotherapy in several types of solid tumors[10-12]. It was defined as the total number of replacement and insert/deletion mutations per mega-base in the exon coding region of the genome examined in tumor samples. It's proposed that tumors with high TMB may produce more neoantigens that can be recognized by the immune system and in elicit broader anti-tumor immune response[13]. Consistently, melanoma patients with higher TMB tumors manifested better clinical response to immune checkpoint inhibitors and long-term survival[14]. However, the clinical application of TMB as a prognostic marker was both time and financial consuming, and the predictive significance of TMB has been questioned by some other studies $[15,16]$. Therefore, there is an urgent need for better biomarkers to predict the effects of immunotherapy on OC.

Genome-wide gene expression profiling provides an extremely valuable tool to unwind complicated biological processes. In this study, we sought to identify prognostic biomarkers for $\mathrm{OC}$ by an integrated TMB and TIME analysis using the $O C$ expression profile data and mutation annotation files downloaded from The Cancer Genome Atlas (TCGA) database. All OC cases were classed into TMB ${ }^{\text {high }}$ group and $\mathrm{TMB}^{\text {low }}$ group based on the OC's somatic mutation data (see Materials and methods). Additionally, we also used ssGSEA (Single Sample Gene Set Enrichment Analysis) scores of immune cell types to conduct unsupervised clustering of $\mathrm{OC}$ patients by the profiles of tumor-infiltrating leukocytes (TILs) and obtained three different immune infiltrating clusters. Samples with higher immune cell infiltration are categorized as the immunity ${ }^{\text {high }}$ group. We further compared up-regulated genes in either TMB ${ }^{\text {high }}$ or immunity ${ }^{\text {high }}$ group and found that 14 genes overlapped in these two subgroups. Survival analysis showed that CXCL13, FCRLA, PLA2G2D, and MS4A1 were significantly correlated with better prognosis. Data of a published melanoma cohort also suggested that these four genes were associated with better response to immune checkpoint blockade-based immunotherapy. Altogether, our study is beneficial to predict the prognosis of $\mathrm{OC}$ patients and provides a simple and rapid method to screen patients for immunotherapy. Our analysis process was shown in Fig. 1.

\section{Methods}

\section{Criteria of the $\mathrm{TMB}^{\text {high }}$ and the $\mathrm{TMB}^{\text {low }}$ Subtypes of Ovarian Cancer}

The TMB score of a tumor sample was calculated as follows[17]:

total number of truncating mutations * $2.0+$ total number of non-truncating mutations * 1.0. Nonsense, frame-shift deletion or insertion, and splice-site mutations were included in the truncating mutation category, and missense, in-frame deletion or insertion, and nonstop mutations were included in the nontruncating mutation category. The TMB ${ }^{\text {high }}$ subtype (samples with TMB scores higher than the third quartile value, $n=86$ ) and the $T_{M B}{ }^{\text {low }}$ subtype (samples with TMB scores lower than the first quartile 
value, $n=86$ ) were defined in ovarian cancer based on the TMB scores of its tumor samples. Then we used the log-rank test to analyze and compare the Kaplan-Meier survival curves of the patients' overall survival (OS).

\section{Criteria of the Immunity high and the Immunity low Subtypes of Ovarian Cancer}

We downloaded 29 immune gene sets for reference (Table S1) and used the "GSVA" R statistical software environment to perform single-sample GSEA (sSGSEA) analysis on OC samples. The ssGSEA algorithm was based on the expression information of specific marker genes of immune cells, which were obtained from the article published by Bindea et al.[18]. Basically, the ssGSEA score was first calculated in terms of different biological functions. Then, the correlations between these functions and the risk scores of samples were analyzed. The immunity ${ }^{\text {high }}$ subtype $(n=193)$ and the immunity low subtype $(n=149)$ were defined in each cancer type based on the comprehensive sSGSEA that derived from the abundance of 29 immune subsets. Finally, the overall survival of patients in each category was analyzed using the log-rank test presented as the Kaplan-Meier survival curves.

\section{Comparisons of Expression Levels of Genes and Gene-sets between two Classes of Samples}

We downloaded RNA-Seq gene expression profiles, gene somatic mutations, and clinical data for ovarian cancer (OC) from the TCGA data portal (https://portal.gdc.cancer.gov/) on April 20, 2019. The TCGA RNASeq gene expression data were normalized by base-2 log transformation. We identified the differential expression genes (DEGs) were identified using the "limma, version = 3.8" package in R software. The student's t-test was used to compare the expression levels of a single gene between two groups of samples. Then we performed Spearman's correlation analysis was performed to clarify the relationship between TMB and immunity.

\section{Tumor Purity Estimation Analysis and Identification of Up-regulated differentially expressed genes (Up- DEGs)}

The "estimate" R package was used to estimate tumor purity, while the "limma" package was applied for data normalization and gene differential expression matrix acquisition. We used the "DESeq2" R package to analyze DEGs. Top 200 DEGs that are up-regulated in the $\mathrm{TMB}^{\text {high }}$ group compared to $\mathrm{TMB}^{\text {low }}$ group were selected as the research targets. DEGs that are up-regulated in immune ${ }^{\text {high }}$ were analyzed through the "limma, version = 3.8" R package. The candidate DEGs were screened by overlapping the up-regulated genes in $\mathrm{TMB}^{\text {high }}$ group and immunity ${ }^{\text {high }}$ group scores. $P$-value $<0.05$, and $\| \log _{2}$ fold change $(\mathrm{FC}) \mid \geq 1$ were set as the cutoff criteria to screen for significant DEGs of interest.

\section{Functional Annotation of DEGs}

The Gene Ontology (GO) enrichment analysis of DEGs screened by the TMB ${ }^{\text {high }}$ group and the immunity high group ("H" vs. "L") was further analyzed using the "org. Hs.eg.db" package, "clusterprofile" package and "enrichplot" package in R software. To explore the potential function of the hub genes in OC, 
we performed GSEA of the DEGs' data. The enrichment scores of molecular pathways and gene expression signatures were evaluated by a single-sample gene set enrichment analysis, and the differences were considered as statistically significant with if $p<0.05$.

\section{GSEA and PPI network Construction of Prognosis-related DEGs}

Gene Set Enrichment Analysis (GSEA) of prognosis-related DEGs was performed using GSEA 3.0 software with gene set c2 (cp.kegg.v.6.2.symbols.gmt). The data being analyzed comes from the RNA expression of 379 ovarian cancer samples in TCGA. Gene expression greater than the median is defined as "High group" in OC patient samples and gene expression greater than the median is defined as "Low group" in OC patient samples. The number and type of permutations in the software were set at "1000" and "phenotype", respectively. $P<0.05$ were regarded as statistically significant.

Protein-protein interactions (PPI) analysis was conducted to reveal the molecular mechanisms of the 14 Up-DEGs in ovarian cancer. We utilized the Search Tool for the Retrieval of Interacting Genes (STRING) protein database 11.0 (http://string-db.org/) to construct the PPI networks. An interaction score $>0.4$ was set as the cut-off criterion. Hiding the disconnected nodes in the network, only the protein interaction network of 9 Up-DEGs was shown.

\section{Exploration of the Correlation Between Pathways and DEGs}

The molecular signatures dataset used for KEGG pathway enrichment analysis is "c2.cp.kegg.v6.2.symbols.gmt" files downloaded from the GSEA website (https://www.gseamsigdb.org/gsea/msigdb/index.jsp). Gene Set Variation Analysis (GSVA) was utilized to select the differential expression Kyoto Encyclopedia of Genes and Genomes (KEGG) pathways in OC[19]. The "GSVA" and "limma" R packages were used to predict significantly different KEGG pathways. Then, unsupervised hierarchical clustering of these top KEGG pathways was performed to distinguish between normal ovaries group (GTEx datasets) and OC (TCGA datasets). KEGG pathway analysis was performed with the standard of $p<0.05, \log _{2}$ fold change $(F C) \mid \geq 0$.2. Heatmaps and clustering were generated using the $\mathrm{R}$ package "pheatmap".

\section{Evaluation of Tumor Microenvironment Infiltration Patterns}

CIBERSORT (https://cibersort.stanford.edu/) was applied to estimate the abundances of different immune cells. LM22 gene signatures were used as references for 22 human immune cell phenotypes, including two $\mathrm{B}$ cell subsets, three $\mathrm{CD} 4^{+} \mathrm{T}$ cell subsets, $\mathrm{CD} 8^{+} \mathrm{T}$ cells, $\mathrm{T}$ cells follicular helper, $\mathrm{T}$ cells

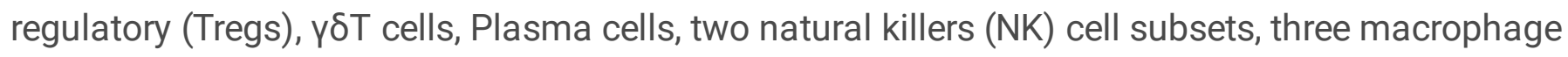
subsets, two dendritic cells (DC) subsets, two Mast cells, monocytes, neutrophils, and eosinophils. We used $\mathrm{R}$ programming ( $\mathrm{R}$ version 3.5.2.) to perform all calculations and statistical analysis.

\section{Construction of Prognostic Analysis of DEGs Signature for Ovarian Cancer}


Kaplan-Meier was used to analyze overall survival. Taking median DEGs expressions as the cut-off point, we divided all patients into a low expression group and a high expression group. The log-rank test was applied to analyzing and comparing the survival curves.

\section{Validation of the Four DEGs Signature}

To validate the relationship between DEGs and patient survival, we use the OC sample from GEO database as the validation set, including GSE30161 ( $n=58)$, GSE9891 ( $n=285)$, GSE63885 ( $n=101)$, GSE26712 ( $n=195)$, GSE15622 ( $n=69)$, GSE19829 ( $n=70)$, GSE18520 ( $n=63)$, GSE26193 $(n=107)$, GSE27651 ( $n=49)$, and TCGA ( $n=427)$. Then, we used multivariate Cox regression analysis to calculate the patient's risk score in the training set. Finally, the "forestplot" R packages were used to draw forest plot.

\section{Estimation of Immune Cells Infiltration}

Tumor immune Estimation Resource (TIMER, https://cistrome.shinyapps.io/timer/) was used to estimate the correlation between 4 DEGs and the immune cell infiltration levels (purity, B cells, CD4 ${ }^{+} \mathrm{T}$ cells, CD8 ${ }^{+} \mathrm{T}$

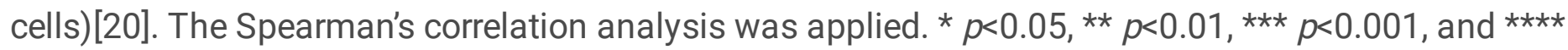
$p<0.0001$.

\section{Prediction of Immune Functions and Immunotherapy of the Four DEGs}

We used tumor immune dysfunction and rejection (TIDE, http://tide.dfci.harvard.edu) algorithm to test the correlation between genetic markers and immune function. Then, we collected data sets of anti-CTLA4 and anti-PD1 therapies melanoma patients[21] and analyzed the Pearson correlation between gene expression and cytotoxic T lymphocyte (CTL) levels.

\section{Statistical and Computational Analyses}

Kaplan-Meier survival curves were used to show the survival (OS) differences between gene higherexpression-level patients and lower-expression-level patients. We used the log-rank test to calculate the significance of survival-time differences between two classes of patients with a threshold of $p$ value<0.05. All the statistical and computational analyses were performed using the R statistical software environment.

\section{Results}

\section{$\mathrm{TMB}^{\text {high }}$ group and immunity ${ }^{\text {high }}$ group have a better overall survival of OC.}

To explore if a high load of somatic mutation is associated with enhanced tumor immune cell infiltration and result in prolonged overall survival, datasets comprising RNAseq from 379 TCGA tumors and mutation annotation files from 436 TCGA tumors (272 which overlapped with RNAseq data) were obtained from the TCGA Data Portal (https://portal.gdc.cancer.gov/) in March 2019. We evaluated all OC 
cases with complete gene expression data and clinical information in TCGA ( $n=417$, except for the blank values). Based on the somatic mutation data across all the OC cases in this study, calculated TMB scores range from 0.0263 to 6.5260. Interestingly, the TMB scores of OC cases at Grade 3 and Grade 4 were significantly higher than those of Grade 1 and Grade $2 O C$ cases (Fig. 2A, $p=0.048$ ). In addition, a multivariate Cox regression analysis of clinical factors of OC patients includes age, grade, and tumor residual. The results show that high TMB was associated with a good prognosis ( $\mathrm{HR}=0.843, p=0.026)$, while the age of patients and tumor residual was correlated with a poor prognosis. TMB can be used as an independent prognostic indicator for OC patients (Fig. 2B). Then based on the ssGSEA scores of OC patients, we identified three immune clusters by unsupervised clustering, 193 cases were defined as immunity ${ }^{\text {high }}$, while 149 cases were considered as immunity ${ }^{\text {low }}$ by comprehensive infiltration level of all 29 immune subsets (Fig. 2C). Moreover, the PD-L1 expression of the immunity high group is significantly higher than that of immunity ${ }^{\text {low }}$ group (Fig. 2C). Furthermore, the tumor purity in immunity ${ }^{\text {high }}$ group was significantly lower than that in immunity ${ }^{\text {low }}$ group, which verified the reliability of our classification (Fig. 2D).

We further accessed whether there is any association between the TMB or immune infiltration profile and the overall survival. Kaplan-Meier survival curves showed that the overall survival time of $\mathrm{TMB}^{\text {high }}$ or immunity ${ }^{\text {high }}$ group was significantly longer than TMB ${ }^{\text {low }}$ or immunity ${ }^{\text {low }}$ group (Fig. $2 \mathrm{E}, 2.58$ years vs. 1.92 years, $p=0.0295$ in the log-rank test. Figure $2 F, 2.50$ years vs. 2.45 years, $p=0.00082$ in log-rank test). We then downloaded and analyzed the expression of reference genes representing 22 immune cell subgroups from CIBERSORT (see Materials and Methods), and further evaluated the abundance of 22 different immune cell subsets in each of the TMB subgroups or the immune subgroups. The results showed that there were significant differences in the abundance of memory B cells and NK cells between the $\mathrm{TMB}^{\text {high }}$ and $\mathrm{TMB}^{\text {low }}$ subgroups, while there were substantial differences in the abundance of 7 immune cell subgroups between the immunity ${ }^{\text {high }}$ and immunity ${ }^{\text {low }}$ subgroups (Figure S1A and S1B).

\section{Functional enrichment analysis of up-regulated differentially expressed genes (Up-DEGs).}

Tumor mutation burden (TMB) or tumor immune infiltration have been demonstrated as a potential biomarker to predict responses to immunotherapy in several types of solid tumors[14-16]. Considering the close correlation between TMB, or the immune cell infiltration and the immune cell infiltration. We comprehensively compared the upregulated DEGs in the TMB ${ }^{\text {high }}$ and immunity ${ }^{\text {high }}$ group to looking for common differential genes. We reasoned that the genes enriched in both these two subgroups would be involved in important biological functions. We crossed the top 200 genes enriched in the TMB ${ }^{\text {high }}$ group with the 427 genes significantly up-regulated in the immunity ${ }^{\text {high }}$ group through the Venn diagram, and finally got 14 Up-DEGs (Fig. 3A). The expression level of 14 Up-DEGs in each case within the TMB group and the immune group were shown in Figure S2A. Next, we downloaded and analyzed the expression of 14 Up-DEGs in immune cells (including T cells and B cells) and ovarian cancer cells on the CCLE website (https://portals.broadinstitute.org/ccle/about). IGLL1, CXCL13, FCRLA, MS4A1, and PLA2G2D are expressed higher in immune cells than in OV cells (Figure S2B). Interestingly, we also found CCL11, IL27, 
and CXCL13 in the signature genes of the "chemokine receptor (CCR)" in Table S1. CXCL13 was also found in the signature genes of "follicular helper T cells (Tfh)". PLA2G2D was found in the signature genes of "CD8 $8^{+} \mathrm{T}$ cells". MS4A1 found in the signature genes of "tumor-infiltrating lymphocytes (TIL)". Finally, we conducted unsupervised hierarchical clustering of KEGG pathways related to 14 Up-DEGs and found that most of these KEGG pathways were immune-related, such as "Cytokine cytokine receptor interaction", "Chemokine signaling pathway", as well as "Primary immunodeficiency" (Fig. 3B). Moreover, to predict the potential function of the DEGs, we performed the functional enrichment analysis of the 14 Up-DEGs. GO terms and KEGG pathway analysis also demonstrate that the Up-DEGs function in the immune and inflammatory response, cytokine activities, and T cell proliferation (Fig. 3C and 3D). These results indicated that the gene sets shared by both $\mathrm{TMB}^{\text {high }}$ and immune $e^{\text {high }}$ groups are involved in active immune functions.

\section{Protein-protein interactions (PPI) of Up-DEGs}

To understand the interrelationship between the 14 Up-DEGs, we used the Search Tool for the Retrieval of Interacting Gene (STRING) tool to establish a PPI network (Fig. 4A). By counting the number of connected nodes, it shows that CXCL13 6 interconnected proteins, which are the most key links with other members of the module in the network. Furthermore, CXCL13, several key genes associated with T cell proliferation, inflammation, and immune response were located at the center of the module, such as PLA2G2D and FCRLA (Fig. 4B). These results indicated that CXCL13, FCRLA, MS4A1, PLA2G2D, and ADAMDEC1 interact more with other molecules, suggesting that these genes are more likely to be key genes in regulating tumor restricting functions in OCs.

\section{Identification of a gene set correlated with better survival.}

Both $\mathrm{TMB}^{\text {high }}$ group and immunity ${ }^{\text {high }}$ group were correlated with better survival. However, the detection of either TMB or tumor immune profile takes much time and resources, which limits their prevalence in the clinic. Based on our previous analysis, we reasoned that there could be potential prognosis predicting biomarkers among the 14 shared Up-DEGs defined above that could be applied to predict the survival. To this end, we analyzed the potential role of Up-DEGs in the overall survival time of OC patients. Among 14 Up-DEGs, four genes, including CXCL13, FCRLA, MS4A1, and PLA2G2D were validated to be significantly relevant to good overall survival and prognosis outcomes (log-rank test, $p<0.05$ ) (Fig. 5A-D). Next, we downloaded from the CPTAC database and analyzed the difference in protein expression of FCRLA and MS4A1 in OC patients with different stages. The results showed that the protein expression of FCRLA and MS4A1as the stage of tumors increase (Figure S3A, B). Furthermore, We analyzed the prognosis of CXCL13, FCRLA, MS4A1, and PLA2G2D on the Human Protein Atlas (HPA, https://www.proteinatlas.org) website[22]. The results showed that the high expression of CXCL13, FCRLA, MS4A1, and PLA2G2D were correlated with a good prognosis of OC (Figure S3C-F). To validate whether the four genes have an equal prognostic value in other OC studies, we downloaded and analyzed the expression datasets for different cohort of the four genes from the GEO database, including GSE30161 ( $n=58)$, GSE9891 $(n=285)$, GSE63885 ( $n=101), \operatorname{GSE} 26712(n=195), \operatorname{GSE} 15622(n=69), \operatorname{GSE} 19829(n=70), \operatorname{GSE} 18520(n=63)$, 
GSE26193 ( $n=107)$, GSE27651 ( $n=49)$. Meta-analysis further indicated that OC cases with high expression of these four genes showed a significant survival benefit (Fig. 5E-H). These results indicated that the transcription levels of CXCL13, FCRLA, MS4A1, and PLA2G2D could be applied to predict the prognosis of $\mathrm{OC}$ patients.

\section{CXCL13, FCRLA, MS4A1, and PLA2G2D correlated with immune status in OC.}

Tumor purity is usually considered when obtaining immunotherapy expression markers from transcriptome data. Some studies have shown that tumor samples with low purity tend to have more immune cells and a higher mutation load[23]. These patients with low purity responded were better to immunotherapy[23]. Therefore, to further validate if the four signature genes can predict tumor immune infiltration, we used TIMER (Tumor Immune Estimation Resource, https://cistrome.shinyapps.io/timer/), an online database, to analyze the infiltration of different immune cells in tumor tissues based on available RNA-seq data. Our results demonstrated that CXCL13, FCRLA, MS4A1, and PLA2G2D have a negative correlation with tumor purity in OC (Fig. 6A-D). Furthermore, CXCL13 expression showed a very weak relationship with $B$ cell infiltration level in OC (Figs. 6A), while FCRLA, MS4A1, PLA2G2D have no significant correlations with B cell infiltration level in OC (Fig. 6B-D). However, there were moderate to strong positive relationships between the expression levels of CXCL13, FCRLA, MS4A1, PLA2G2D, and infiltration level of $\mathrm{CD} 4^{+} \mathrm{T}$ cells, as well as have a prominent positive correlation between expression level and infiltration level of CD8 ${ }^{+}$T cells in OC, especially CXCL13, FCRLA, MS4A1, and PLA2G2D (Figs. 6A-D). More information can be found in Table S2. In conclusion, CXCL13, FCRLA, MS4A1, and PLA2G2D might have multiple and closely function in immune infiltration.

To identify the pathways that these four genes might be involved in gene set enrichment analysis (GSEA) was performed in the published TCGA ovarian cancer database $(n=304)$. The results indicated that all of four genes were related to immune cell signaling pathways. They were all associated with the $B$ cell receptor, chemokine, cytokine-cytokine receptor interaction, natural killer cell-mediated cytotoxicity, and $T$ cell receptor signaling pathways (Fig. 6E-H). Interestingly, CXCL13, FCRLA, and PLA2G2D were also associated with primary immunodeficiency (Fig. 6E-H). MS4A1 was associated with autoimmune thyroid disease (Fig. 6G). All of these findings revealed that CXCL13, FCRLA, MS4A1, and PLA2G2D's expression was closely related to immune function. More information can be found in Table S3. These results indicated that CXCL13, FCRLA, MS4A1, and PLA2G2D were associated with immune pathways.

Altogether, all the results indicated that CXCL13, FCRLA, MS4A1, and PLA2G2D were associated with immune effector cell infiltrations and involved in immune pathways.

\section{CXCL13, FCRLA, MS4A1, and PLA2G2D correlated with immunotherapy.}

Immune infiltration and active inflammations are prerequisites of anti-tumor immune reaction and better response to immunotherapy. We have shown that CXCL13, FCRLA, PLA2G2D, and MS4A1 are upregulated in the $\mathrm{TMB}^{\text {high }}$ group and immunity ${ }^{\text {high }}$ group, involved in immune functions, and are associated with better survival. We then asked if we could use the transcription levels of these four genes 
to predict the response to immunotherapy. Since the data of OC response to immunotherapy are not available, to solve this problem, we explored an online database-TIDE, then analyzed the data collected from the melanoma patients treated with anti-PD-1 or anti-CTLA4 alone from Gide et al. in the TIDE website[21]. Previous reports showed that some tumors have a high level of infiltration by cytotoxic $T$ cells[24, 25]. Then the CTL levels were used in the TIDE website to evaluate the tumor immune infiltration of these four Up-DEGs. As shown in Fig. 7A-D, our results showed that the CXCL13, FCRLA, MS4A1, and PLA2G2D were positively correlated with the level of CTL infiltration in the melanoma patients treated with anti-PD-L1 or anti-CTLA4 (Fig. 7A-D). Moreover, we also found that the high expression levels of CXCL13, FCRLA, MS4A1, and PLA2G2D were positively associated with the better response of melanoma patients treated with anti-PD1 and anti-CTLA4 (Fig. 7E-H). These results indicated that CXCL13, FCRLA, MS4A1, and PLA2G2D could be applied to predict responses to immunotherapies.

\section{Discussion}

Ovarian cancer has been one of the major threats to women's health for decades[26]. Current treatments for late-stage $\mathrm{OC}$ patients are far less satisfying. At present, accumulating clinical data indicated that inflammatory tumors with the high expression level of PD-L1 are more likely to respond[27]. Immune checkpoint blockade based immunotherapy, especially PD-1/PD-L1 blocking antibody, has been approved for clinical treatment to multiple solid tumors[28-30]. However, the PD-L1 blocking antibody has not been approved for treating OC. The reasons are likely due to scarce information of the TIME of OC, resulting in missing targetable immune-modulatory molecules as well as promising biomarkers for choosing the right patients population for immunotherapy.

TMB represents the overall amount of somatic mutations that could generate neoantigens to elicit immune response[31-34]. Consistently, tumors with high TMB scores contain more TILs and are associated with better response to immunotherapy response[35], such as melanoma[36] and nonsmallcell lung cancer (NSCLC)[37], with high TMB have shown better response and treatment results in immune checkpoint blockade treatment. However, the clinical application of TMB as a prognostic marker was both time and financial consuming, and the predictive significance of TMB has been questioned by some other studies $[15,16]$. Therefore, there is an urgent need for better markers to predict the effects of TMB and immunotherapy. In our study, we combined TMB and TIME evaluation methods to identify a set of genes related to the immune status of ovarian cancer, including CXCL13, FCRLA, MS4A1, and PLA2G2D. The expression of these genes has a better prognosis of OC. In addition, multiple reports have shown that these four genes were involved in immune reactions. For example, CXCL13 has recently been linked with T Follicular helper (TFH) cells infiltration and improved survival[38]. FCRLA has been shown to be a potential target gene in immunotherapy for B-cell lymphoma[39]. MS4A1 played a vital role in the apoptosis of B-cell lymphoma Ramos cells[40]. PLA2G2D involved in inflammation and immune response and may be used to treat inflammatory diseases[41]. The association of these four genes and immune cell infiltration was further validated by an independent algorithm presented in the TIMER database (Fig. 6). However, positive association does not mean these genes are causative factors that affect the tumor inflammatory status, which takes more studies. 
Due to the lack of data on OC's response to immunotherapy, we analyzed the data collected from a melanoma cohort. It was found that in melanoma patients treated with anti-PD-L1 or anti-CTLA4, these four genes were positively correlated with the number of CTL infiltration, and their high expression was associated with a better response of patients to immunotherapy (Fig. 7). Further effort will focus on validating antibodies or developing other diagnostic methods to detecting the expression of these genes in the tumor microenvironment, which allows us to pick the right patients for immune checkpoint blockade based immune therapy against OCs.

In summary, by a comprehensive study of TMB and TIME, we also identified a signature gene set composed of four genes that are associated with the immune status of $\mathrm{OC}$ and could be used as biomarkers to predict overall survival and response to immunotherapy. This gene set could guide future immunotherapy on OC.

\section{Conclusions}

In our study, through an integrated TMB and TIME analysis, four genes, CXCL13, FCRLA, MS4A1, and PLA2G2D were eventually identified as candidate OC biomarkers, which were enriched in signaling pathways closely related to immunity. These four genes were significantly associated with immune infiltration and good prognosis of $\mathrm{OC}$ and are associated with a better response of melanoma to immunotherapy. Further research on these genes may provide new light on the potential relationship between tumor microenvironment and OC prognosis.

\section{Abbreviations}

TMB: Tumor Mutational Burden; Tumor Immune Microenvironment: TIME; OC: Ovarian Cancer; TCGA: The Cancer Genome Atlas; ssGSEA: Single-sample Gene Set Enrichment Analysis; Up-DEGs: Up-regulated Differential Expression Genes; GO: Gene Ontology; KEGG: Kyoto Encyclopedia of Genes and Genomes pathway; PPI: Protein-Protein Interaction; DAVID: Database for Annotation, Visualization, and Integrated Discovery; GSVA: Gene Set Variation Analysis; STRING: Search Tool for the Retrieval of Interacting Genes; DISCOVER: Discrete Independence Statistic Controlling for Observations with Varying Event Rates; GEO: Group on Earth Observations; OS: Overall Survival; TIMER: Tumor Immune Estimation Resource; GSEA: Gene Set Enrichment Analysis; ssGSEA: Single Sample Gene Set Enrichment Analysis; TIDE: Tumor Immune Dysfunction and Exclusion; IHC: immunohistochemistry; CTL: cytotoxic T lymphocyte; Human Protein Atlas: HPA; TFH: T Follicular helper cells.

\section{Declarations}

\section{Ethics approval and consent to participate}

Not Applicable

\section{Consent for publication}


We have obtained consent to publish this paper from all the participants of this research.

\section{Availability of data and materials}

All data generated or analyzed during the study period were included in this published article and its supplementary information.

\section{Competing interests}

The authors declare that they have no competing interests.

\section{Funding}

This work was supported by the National Natural Science Foundation of China [No. 81572900]; The Fundamental Research Funds for the Central Universities of Central South University [No. 502221804]; National Key R\&D Program of China, Stem Cell, and Translation Research [No. 2016YFA0102000]

\section{Authors' contributions}

Lili Fan conceived and designed this study, also crafted figures and tables, and was responsible for the critical reading of the manuscript. Also, she contributed to the data collection and analysis and the critical reading of the manuscript. Han Lei, Ying Lin, and Tianxiang Zhang were responsible for the writing and helped with the figures and tables. Han Lei, Zhengwei Zhou, Zhipeng Yan, Guang Shu, and Gang Yin supervised and contributed to the critical reading of the manuscript.

\section{Acknowledgments}

The authors gratefully acknowledge contributions from the TCGA network, the GTEx network, and the CCLE Network. This study was supported by grants from the National Natural Science Foundation of China (No. 81572900). The Fundamental Research Funds for the Central Universities of Central South University (No. 502221804).

\section{References}

1. Chornokur G, Amankwah EK, Schildkraut JM, Phelan CM: Global ovarian cancer health disparities. Gynecol Oncol 2013, 129:258-264.

2. Menon U, Griffin M, Gentry-Maharaj A: Ovarian cancer screening-current status, future directions. Gynecol Oncol 2014, 132:490-495.

3. Siegel RL, Miller KD, Jemal A: Cancer statistics, 2019. CA Cancer J Clin 2019, 69:7-34.

4. Lee JY, Kim S, Kim YT, Lim MC, Lee B, Jung KW, Kim JW, Park SY, Won YJ: Changes in ovarian cancer survival during the 20 years before the era of targeted therapy. BMC Cancer 2018, 18:601.

5. Hoos A: Development of immuno-oncology drugs - from CTLA4 to PD1 to the next generations. Nat Rev Drug Discov 2016, 15:235-247. 
6. Hamanishi J, Mandai M, Ikeda T, Minami M, Kawaguchi A, Murayama T, Kanai M, Mori Y, Matsumoto S, Chikuma S, et al: Safety and Antitumor Activity of Anti-PD-1 Antibody, Nivolumab, in Patients With Platinum-Resistant Ovarian Cancer. J Clin Oncol 2015, 33:4015-4022.

7. Varga A, Piha-Paul S, Ott PA, Mehnert JM, Berton-Rigaud D, Morosky A, Yang P, Ruman J, Matei D: Pembrolizumab in patients with programmed death ligand 1-positive advanced ovarian cancer: Analysis of KEYNOTE-028. Gynecol Oncol 2019, 152:243-250.

8. Sokratous G, Polyzoidis S, Ashkan K: Immune infiltration of tumor microenvironment following immunotherapy for glioblastoma multiforme. Hum Vaccin Immunother 2017, 13:2575-2582.

9. Hanahan D, Coussens LM: Accessories to the crime: functions of cells recruited to the tumor microenvironment. Cancer Cell 2012, 21:309-322.

10. Goodman AM, Kato S, Bazhenova L, Patel SP, Frampton GM, Miller V, Stephens PJ, Daniels GA, Kurzrock R: Tumor Mutational Burden as an Independent Predictor of Response to Immunotherapy in Diverse Cancers. Mol Cancer Ther 2017, 16:2598-2608.

11. Birkbak NJ, Kochupurakkal B, Izarzugaza JM, Eklund AC, Li Y, Liu J, Szallasi Z, Matulonis UA, Richardson AL, Iglehart JD, Wang ZC: Tumor mutation burden forecasts outcome in ovarian cancer with BRCA1 or BRCA2 mutations. PLoS One 2013, 8:e80023.

12. Duffy MJ, Crown J: Biomarkers for Predicting Response to Immunotherapy with Immune Checkpoint Inhibitors in Cancer Patients. Clin Chem 2019, 65:1228-1238.

13. McGranahan N, Furness AJ, Rosenthal R, Ramskov S, Lyngaa R, Saini SK, Jamal-Hanjani M, Wilson GA, Birkbak NJ, Hiley CT, et al: Clonal neoantigens elicit T cell immunoreactivity and sensitivity to immune checkpoint blockade. Science 2016, 351:1463-1469.

14. Samstein RM, Lee CH, Shoushtari AN, Hellmann MD, Shen R, Janjigian YY, Barron DA, Zehir A, Jordan EJ, Omuro A, et al: Tumor mutational load predicts survival after immunotherapy across multiple cancer types. Nat Genet 2019, 51:202-206.

15. Garassino M, Rodriguez-Abreu D, Gadgeel S, Esteban E, Felip E, Speranza G, Reck M, Hui R, Boyer M, Cristescu R, et al: Evaluation of TMB in KEYNOTE-189: Pembrolizumab Plus Chemotherapy vs Placebo Plus Chemotherapy for Nonsquamous NSCLC. Journal of Thoracic Oncology 2019, 14:S216-S217.

16. Langer C, Gadgeel S, Borghaei H, Patnaik A, Powell S, Gentzler R, Yang JC, Gubens M, Sequist L, Awad M, et al: KEYNOTE-021: TMB and Outcomes for Carboplatin and Pemetrexed With or Without Pembrolizumab for Nonsquamous NSCLC. Journal of Thoracic Oncology 2019, 14:S216-S216.

17. Wang $\mathrm{X}$, Li M: Correlate tumor mutation burden with immune signatures in human cancers. $B M C$ Immunol 2019, 20:4.

18. Bindea G, Mlecnik B, Tosolini M, Kirilovsky A, Waldner M, Obenauf AC, Angell H, Fredriksen T, Lafontaine L, Berger A, et al: Spatiotemporal dynamics of intratumoral immune cells reveal the immune landscape in human cancer. Immunity 2013, 39:782-795.

19. Hänzelmann S, Castelo R, Guinney J: GSVA: gene set variation analysis for microarray and RNA-seq data. BMC Bioinformatics 2013, 14:7. 
20. Li T, Fan J, Wang B, Traugh N, Chen Q, Liu JS, Li B, Liu XS: TIMER: A Web Server for Comprehensive Analysis of Tumor-Infiltrating Immune Cells. Cancer Res 2017, 77:e108-e110.

21. Gide TN, Quek C, Menzies AM, Tasker AT, Shang P, Holst J, Madore J, Lim SY, Velickovic R, Wongchenko $\mathrm{M}$, et al: Distinct Immune Cell Populations Define Response to Anti-PD-1 Monotherapy and Anti-PD-1/Anti-CTLA-4 Combined Therapy. Cancer Cel/ 2019, 35:238-255.e236.

22. Uhlén M, Fagerberg L, Hallström BM, Lindskog C, Oksvold P, Mardinoglu A, Sivertsson A, Kampf C, Sjöstedt E, Asplund A, et al: Proteomics. Tissue-based map of the human proteome. Science 2015, 347:1260419.

23. Aran D, Sirota M, Butte AJ: Systematic pan-cancer analysis of tumour purity. Nat Commun 2015, 6:8971.

24. Wherry EJ, Kurachi M: Molecular and cellular insights into T cell exhaustion. Nat Rev Immuno/ 2015, 15:486-499.

25. Jiang P, Gu S, Pan D, Fu J, Sahu A, Hu X, Li Z, Traugh N, Bu X, Li B, et al: Signatures of T cell dysfunction and exclusion predict cancer immunotherapy response. Nat Med 2018, 24:1550-1558.

26. Siegel RL, Miller KD, Jemal A: Cancer statistics, 2016. CA Cancer J Clin 2016, 66:7-30.

27. Bast RC, Jr., Hennessy B, Mills GB: The biology of ovarian cancer: new opportunities for translation. Nat Rev Cancer 2009, 9:415-428.

28. Radziewicz H, Ibegbu CC, Fernandez ML, Workowski KA, Obideen K, Wehbi M, Hanson HL, Steinberg $J P$, Masopust $D$, Wherry EJ, et al: Liver-infiltrating lymphocytes in chronic human hepatitis $C$ virus infection display an exhausted phenotype with high levels of PD-1 and low levels of CD127 expression. J Virol 2007, 81:2545-2553.

29. Hirano F, Kaneko K, Tamura H, Dong H, Wang S, Ichikawa M, Rietz C, Flies DB, Lau JS, Zhu G, et al: Blockade of B7-H1 and PD-1 by monoclonal antibodies potentiates cancer therapeutic immunity. Cancer Res 2005, 65:1089-1096.

30. Rizvi NA, Hellmann MD, Snyder A, Kvistborg P, Makarov V, Havel JJ, Lee W, Yuan J, Wong P, Ho TS, et al: Cancer immunology. Mutational landscape determines sensitivity to PD-1 blockade in non-small cell lung cancer. Science 2015, 348:124-128.

31. Bracarda S, Altavilla A, Hamzaj A, Sisani M, Marrocolo F, Del Buono S, Danielli R: Immunologic checkpoints blockade in renal cell, prostate, and urothelial malignancies. Semin Oncol 2015, 42:495505.

32. Nadal E, Massuti B, Dómine M, García-Campelo R, Cobo M, Felip E: Immunotherapy with checkpoint inhibitors in non-small cell lung cancer: insights from long-term survivors. Cancer Immunol Immunother 2019, 68:341-352.

33. Chan TA, Yarchoan M, Jaffee E, Swanton C, Quezada SA, Stenzinger A, Peters S: Development of tumor mutation burden as an immunotherapy biomarker: utility for the oncology clinic. Ann Oncol 2019, 30:44-56.

34. Gubin MM, Zhang X, Schuster H, Caron E, Ward JP, Noguchi T, Ivanova Y, Hundal J, Arthur CD, Krebber WJ, et al: Checkpoint blockade cancer immunotherapy targets tumour-specific mutant antigens. 
Nature 2014, 515:577-581.

35. Klebanov N, Artomov M, Goggins WB, Daly E, Daly MJ, Tsao H: Burden of unique and low prevalence somatic mutations correlates with cancer survival. Sci Rep 2019, 9:4848.

36. Valsecchi ME: Combined Nivolumab and Ipilimumab or Monotherapy in Untreated Melanoma. N Engl J Med 2015, 373:1270.

37. Herbst RS, Baas P, Kim DW, Felip E, Pérez-Gracia JL, Han JY, Molina J, Kim JH, Arvis CD, Ahn MJ, et al: Pembrolizumab versus docetaxel for previously treated, PD-L1-positive, advanced non-small-cell lung cancer (KEYNOTE-010): a randomised controlled trial. Lancet 2016, 387:1540-1550.

38. Gu-Trantien C, Migliori E, Buisseret L, de Wind A, Brohée S, Garaud S, Noël G, Dang Chi VL, Lodewyckx JN, Naveaux C, et al: CXCL13-producing TFH cells link immune suppression and adaptive memory in human breast cancer. JCl Insight 2017, 2.

39. Inozume T, Mitsui H, Okamoto T, Matsuzaki Y, Kawakami Y, Shibagaki N, Shimada S: Dendritic cells transduced with autoantigen FCRLA induce cytotoxic lymphocytes and vaccinate against murine Bcell lymphoma. J Invest Dermatol 2007, 127:2818-2822.

40. Kawabata KC, Ehata S, Komuro A, Takeuchi K, Miyazono K: TGF- $\beta$-induced apoptosis of B-cell lymphoma Ramos cells through reduction of MS4A1/CD20. Oncogene 2013, 32:2096-2106.

41. Miki Y, Yamamoto K, Taketomi Y, Sato H, Shimo K, Kobayashi T, Ishikawa Y, Ishii T, Nakanishi H, Ikeda $\mathrm{K}$, et al: Lymphoid tissue phospholipase $\mathbf{A} 2$ group IID resolves contact hypersensitivity by driving antiinflammatory lipid mediators. J Exp Med 2013, 210:1217-1234.

\section{Figures}




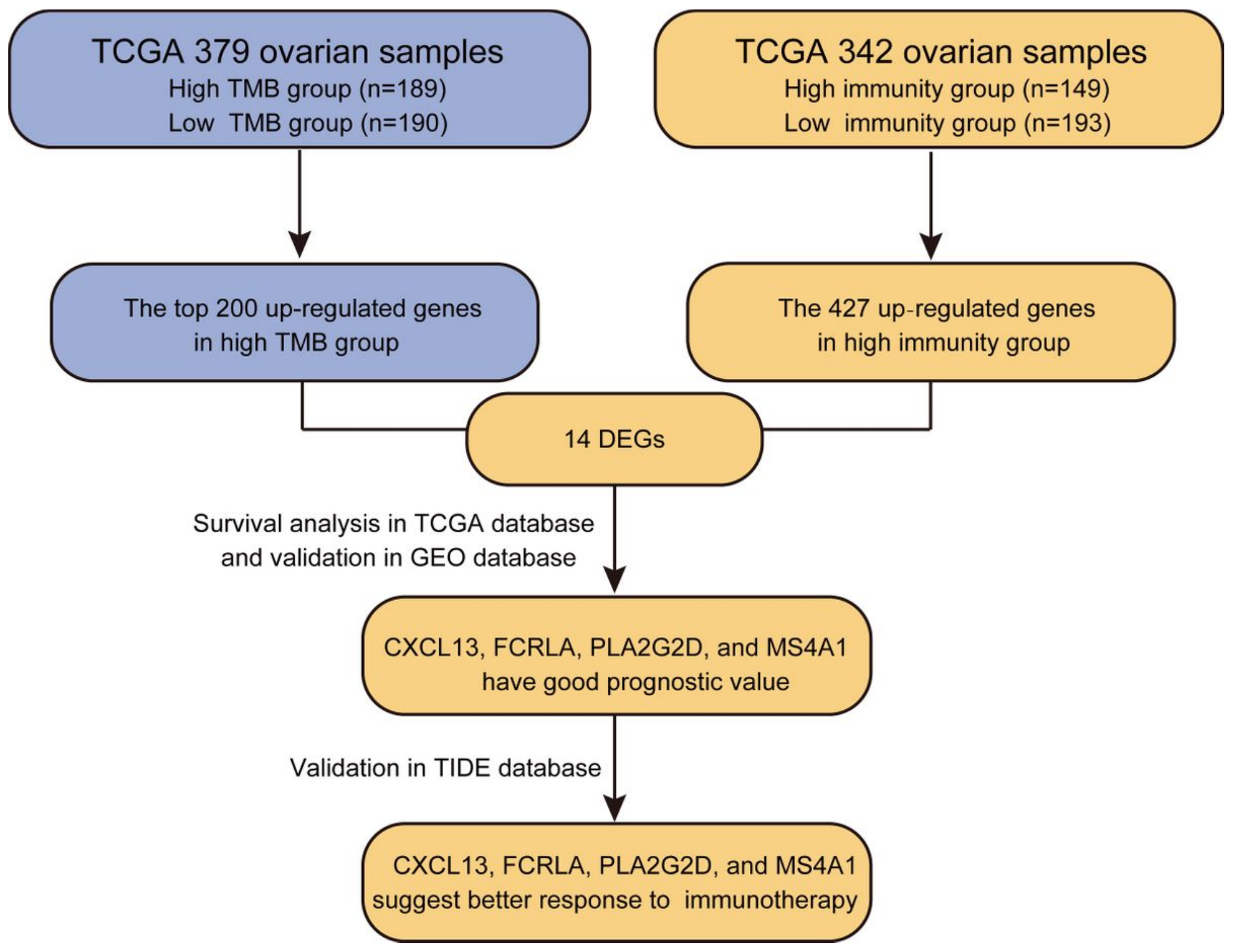

Figure 1

The workflow of the current work. 
A
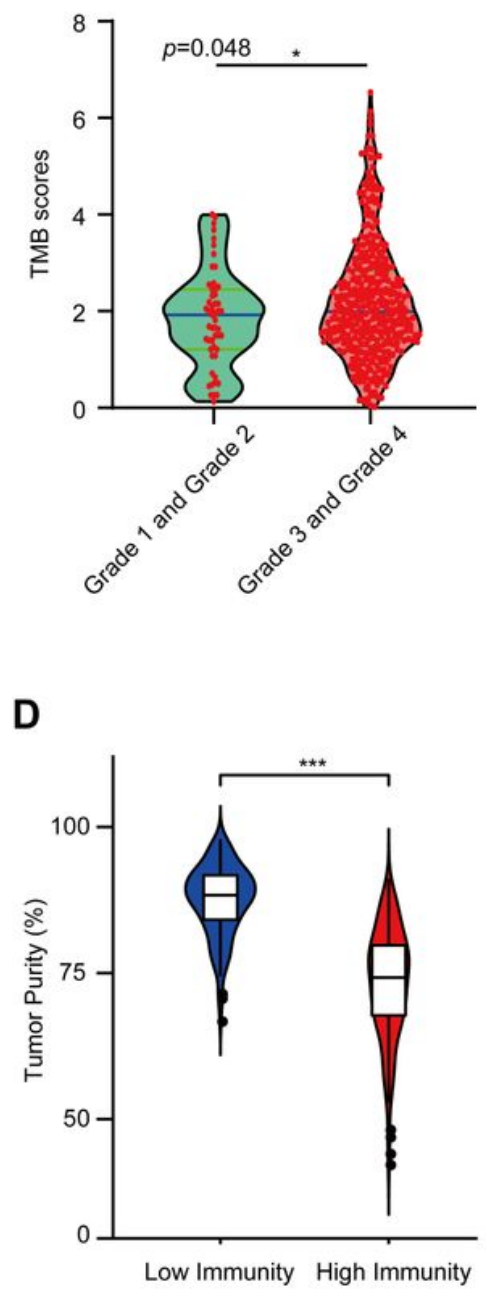

B
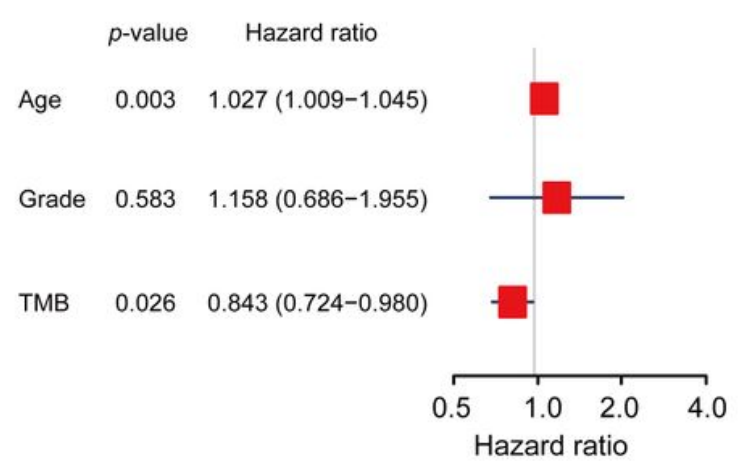

E

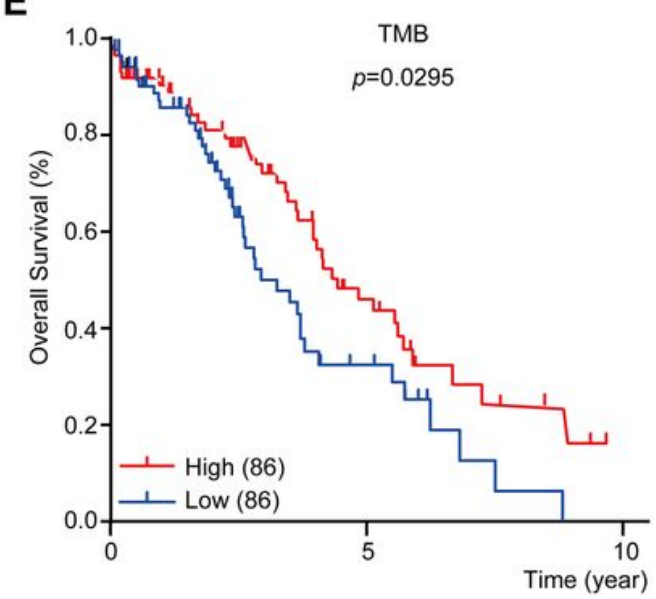

C
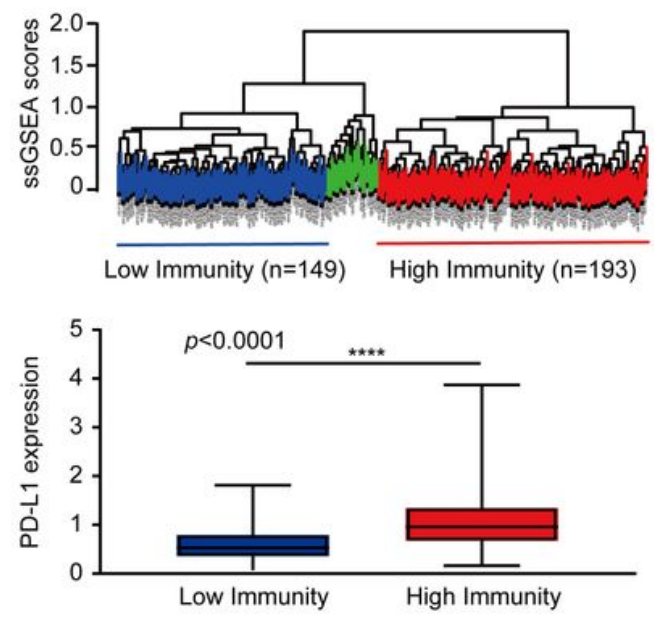

$\mathbf{F}$

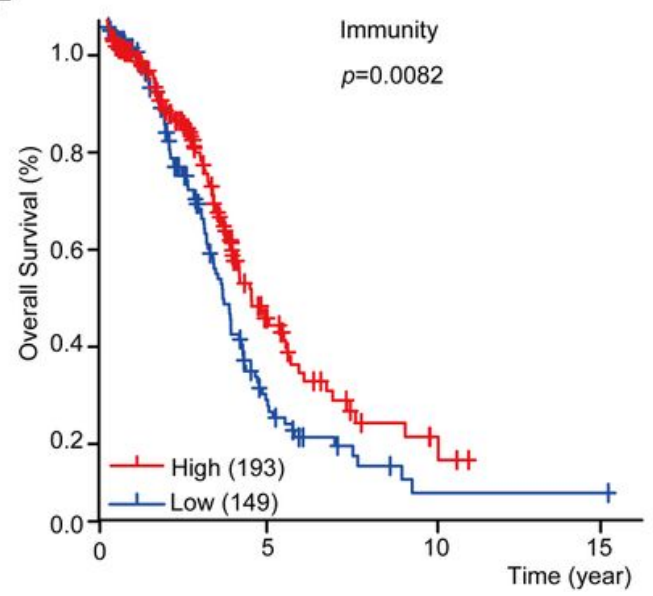

Figure 2

TMBhigh group and immunityhigh group have a better overall survival of OC. (A) The violin plots showed the interrelationship between TMB scores and OC grades ( $n=417$, except for the blank values, $p=0.0480)$. (B) Multivariate Cox regression analysis analyzes TMB and OC patient clinical factors. (C) Up: Unsupervised clustering of OC patients from the TCGA cohort using sSGSEA scores from immune cell types. Hierarchical clustering was performed with Euclidean distance and Ward linkage. Three distinct immune infiltration clusters, here we defined immunitylow group (blue) and immunityhigh group (red), respectively. Down: Box plot showed that PD-L1 expression was significantly higher in immunityhigh group (As displayed by Mann-Whitney test, $\mathrm{p}<0.0001$ ). (D) The violin box plot showed that the tumor purity in immunityhigh group is significantly lower (log-rank test, $p<0.05$ ). (E) OC patients were classified into two groups based on TMB scores: the samples with TMBhigh scores in the top $20 \%$ and the TMBlow samples with TMB scores in the bottom $20 \%$ of all OC samples. According to the Kaplan-Meier survival curve, the overall survival time of TMBhigh group is longer than TMBlow group (2.58 years vs. 1.92 years, $\mathrm{n}=430$, except for the blank values, as displayed by the long-rank test, $\mathrm{p}=0.0295)$. (F) OC patients were classified into two groups based on ssGSEA scores: those with immunityhigh group in 193 patients and those with immunitylow group in 149 patients. The overall survival time of immunityhigh group is longer 
than immunitylow group (2.77 years vs. 2.67 years, $n=364$, except for the blank values, as displayed by the log-rank test, $p=0.0082)$.

\section{A}

The top 200 upregulated

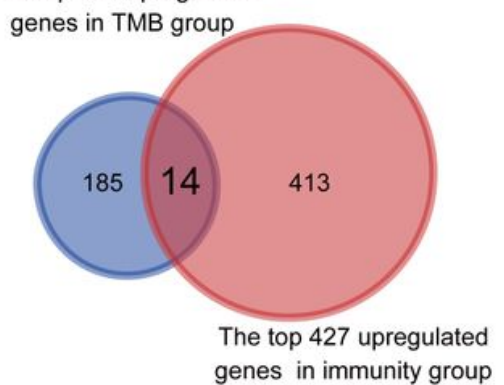

B

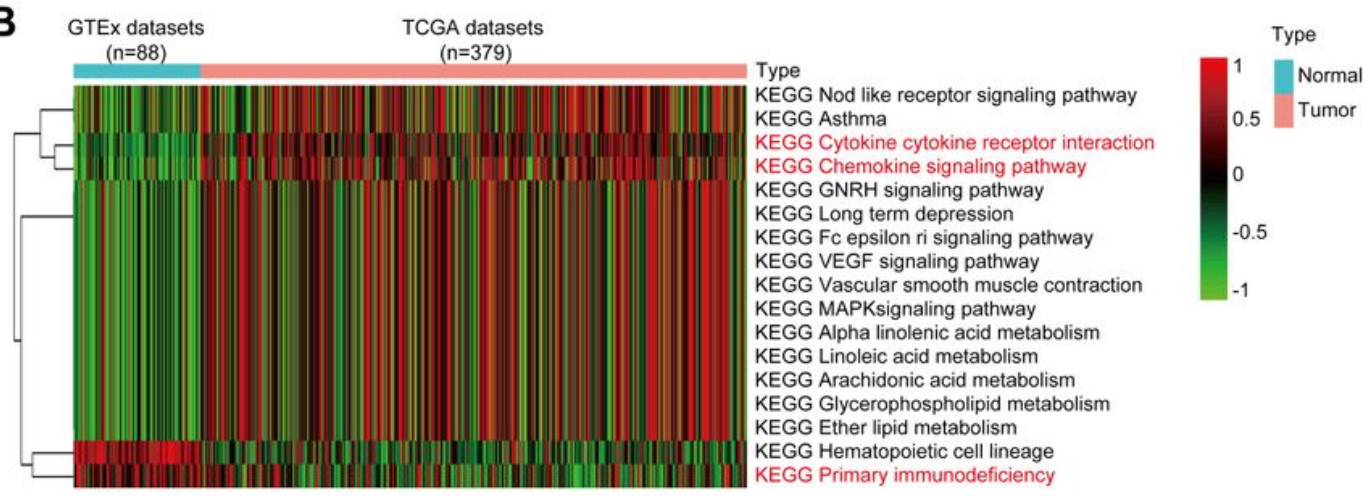

D

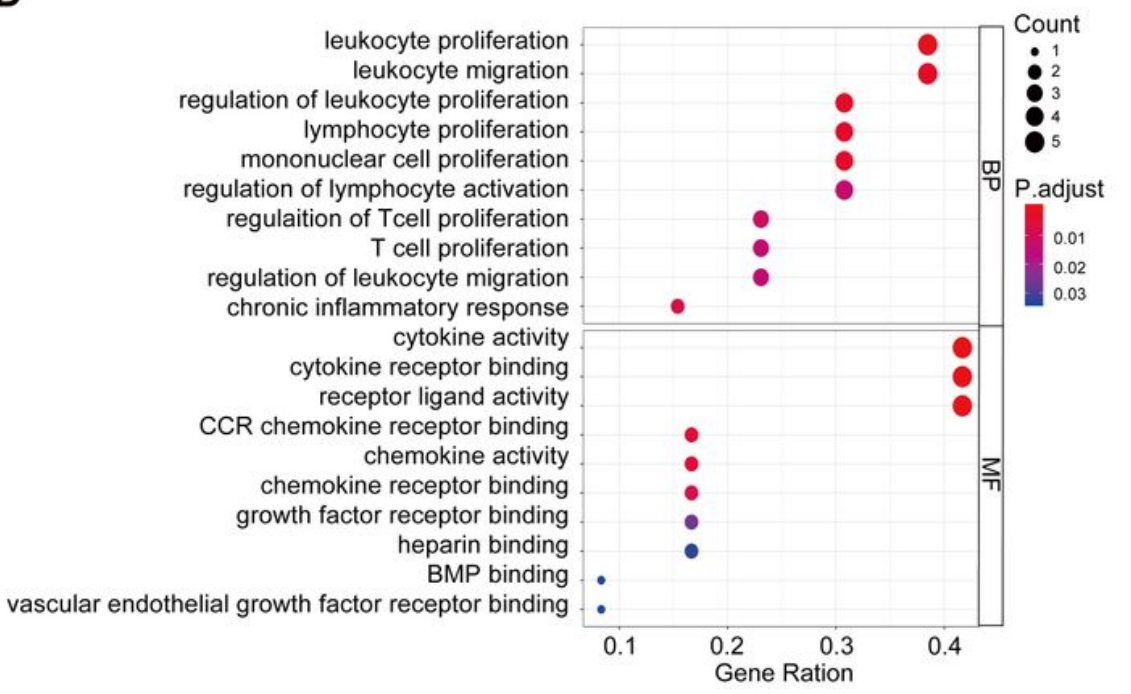

E

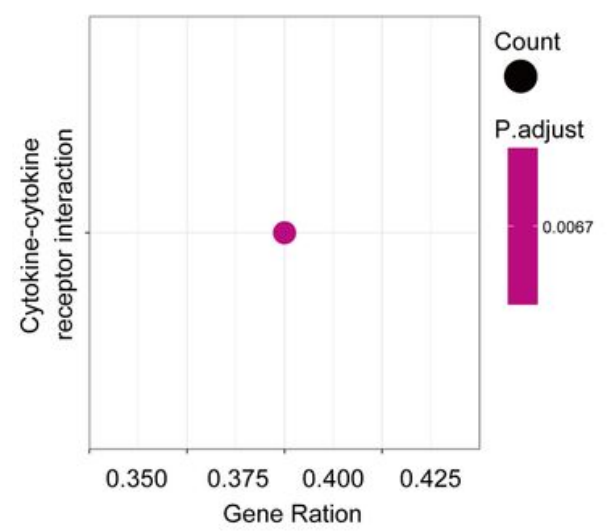

\section{Figure 3}

KEGG pathway and GO term analysis of Up-DEGs. (A) Venn diagrams displayed the amount of conjointly Up-DEGs in both TMBhigh group and immunityhigh group. (B) Unsupervised hierarchical clustering of KEGG pathways associated with 14 Up-DEGs. Blue represents normal samples, data from GTEx ( $n=88)$, red represents OC samples from the TCGA data set(n=379). The average linkage method and the Pearson distance measurement method were used to depict the Heatmaps. High expression genes are shown in red, low expression genes in green, and genes with the same level of expression are shown in black. False discovery rate $(F D R)<0.05$, fold change>1. (C, D) Enriched GO and KEGG pathways of 14 Up-DEGs by using $\mathrm{R}$ software. The $\mathrm{X}$-axis serves as the proportion of relevant genes, and the $\mathrm{Y}$-axis serves as the $\mathrm{GO}$ and KEGG terms. Each bubble means a term. The size of the bubble implies the account of relevant genes. Lighter colors suggest the smaller $\mathrm{p}$ values. C: Enriched GO terms. D: Enriched KEGG terms. FDR of GO and KEGG pathway analysis was acquired from the DAVID functional annotation tool. $P<0.05$. 
A

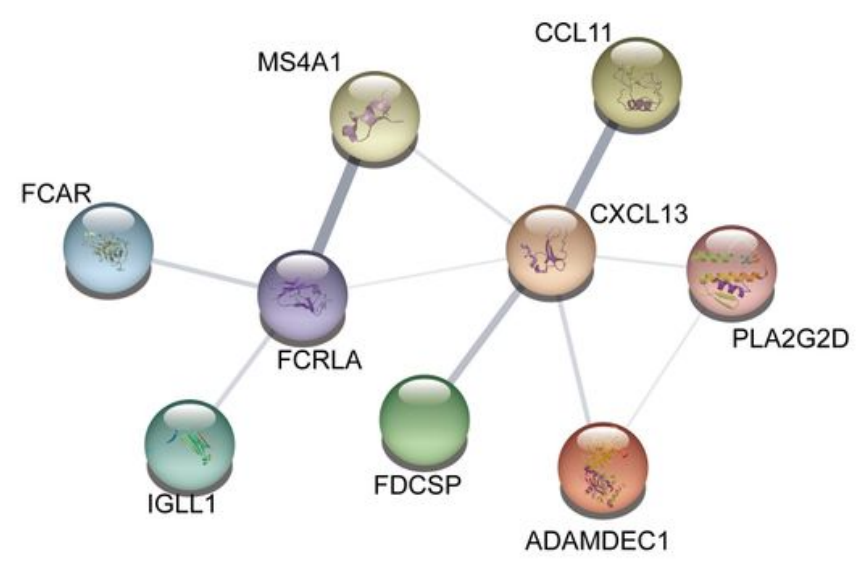

B

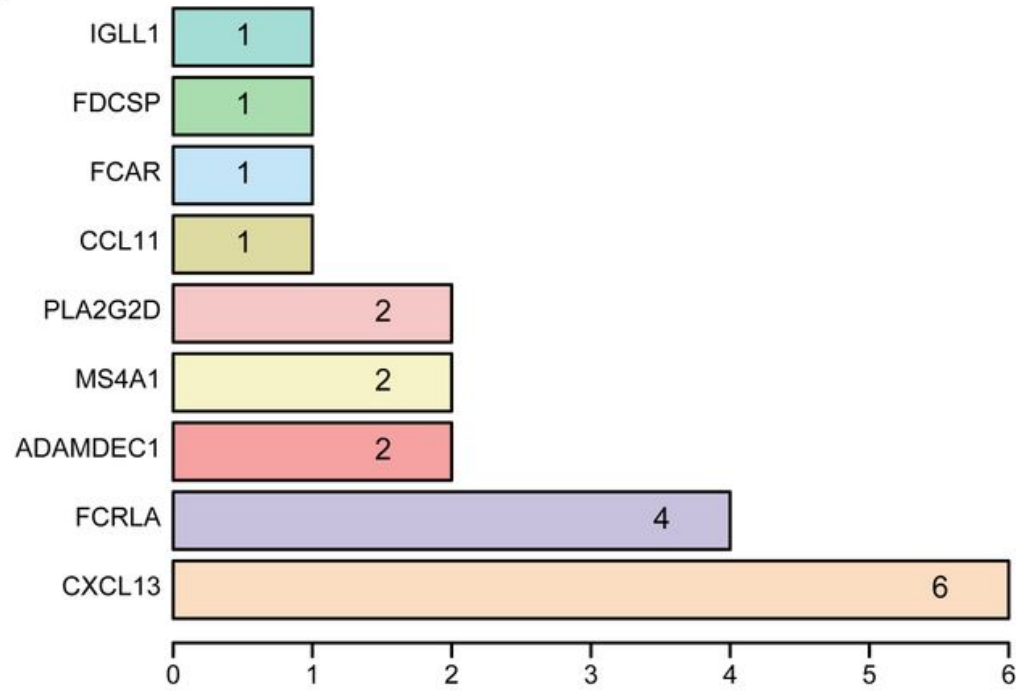

\section{Figure 4}

PPI networks of the Up-DEGs Module. (A) PPI network established by the STRING database for the UpDEGs module. (B) Quantification diagram of the Up-DEGs module. 
A

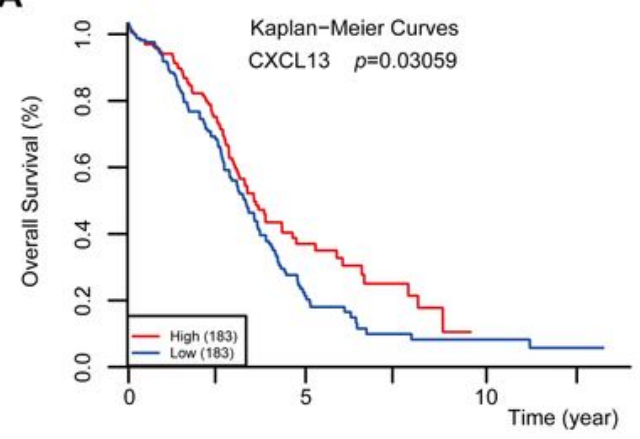

C

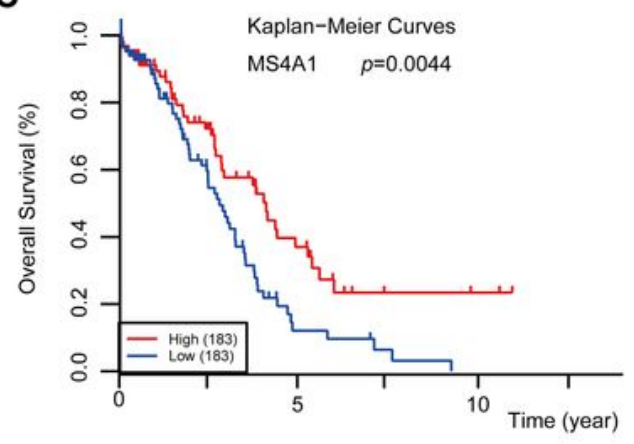

E

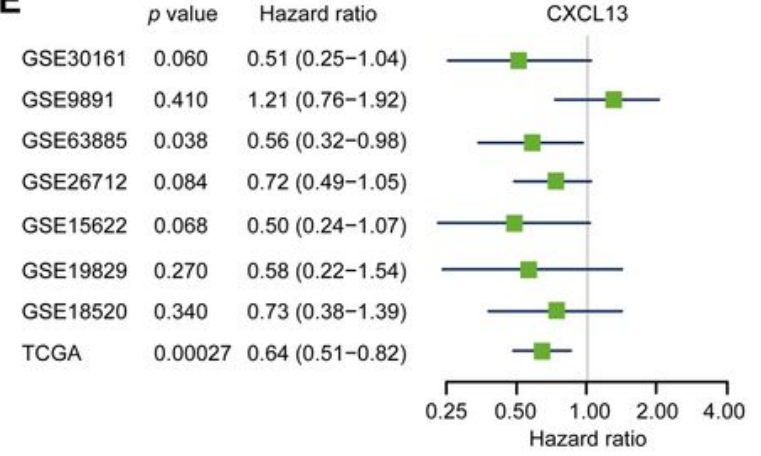

G

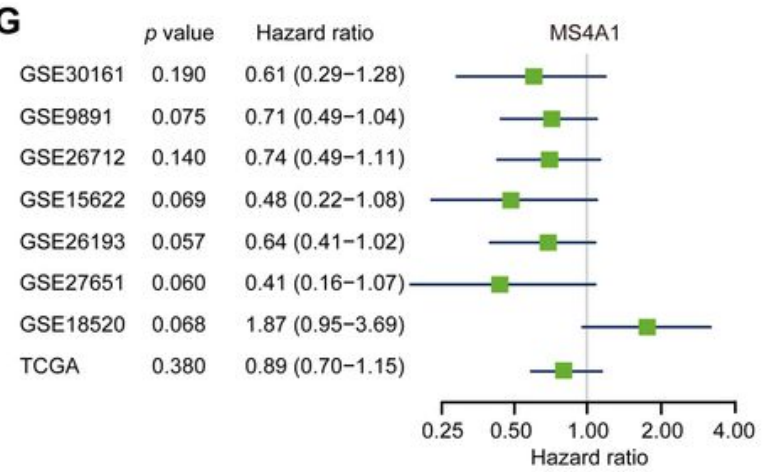

B

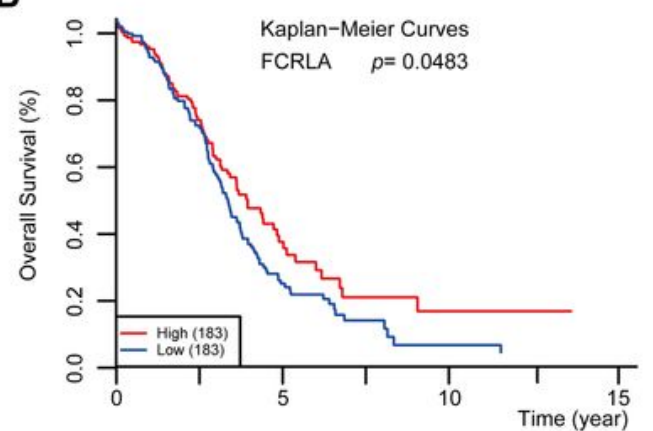

D

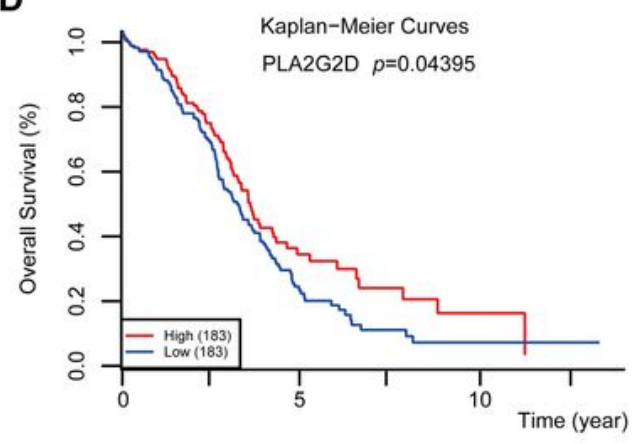

F

F $p$ value Hazard ratio $\quad$ FCRLA

GSE30161 $0.029 \quad 0.47(0.23-0.94)$

GSE9891 $0.0019 \quad 0.51(0.33-0.79) \quad-\square-$

GSE63885 $0.180 \quad 0.69(0.40-1.20)$

GSE19829 $0.320 \quad 0.61(0.22-1.65)$

GSE18520 $0.120 \quad 0.61(0.32-1.15)$

GSE26193 $0.15 \quad 1.39(0.89-2.19)$

GSE27651 $0.48 \quad 1.36(0.57-3.26)$

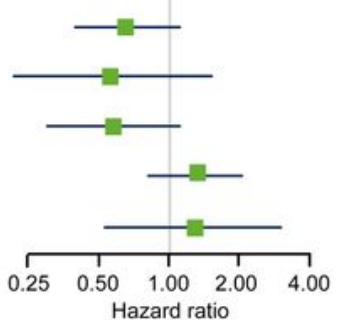

H

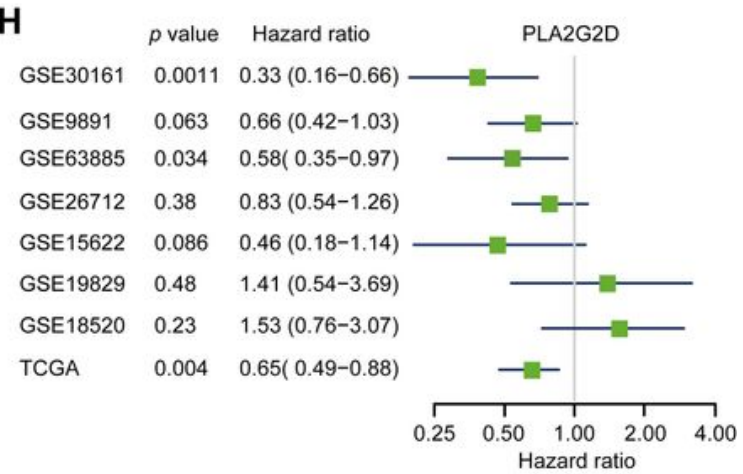

\section{Figure 5}

CXCL13, FCRLA, MS4A1, and PLA2G2D were correlated with a good prognosis of OC. (A-D) Kaplan-Meier curves for overall survival probability in 366 OC patients with low $(n=183)$ and high (183) CXCL13, FCRLA, MS4A1, and PLA2G2D expression (analyzed with log-rank test, $\mathrm{p}<0.05$ ). (E-H) Meta-analysis describing forest plots of CXCL13, FCRLA, MS4A1, and PLA2G2D expression as a univariate predictor of overall survival. 
A
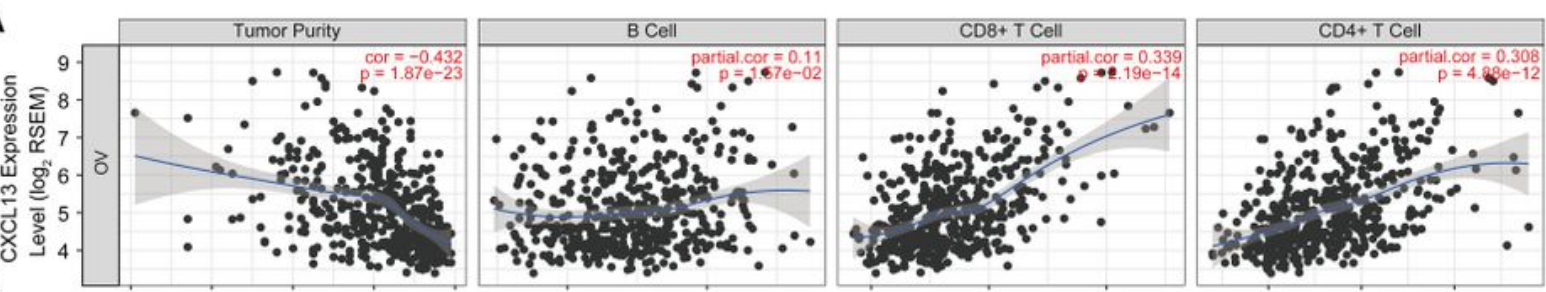

B
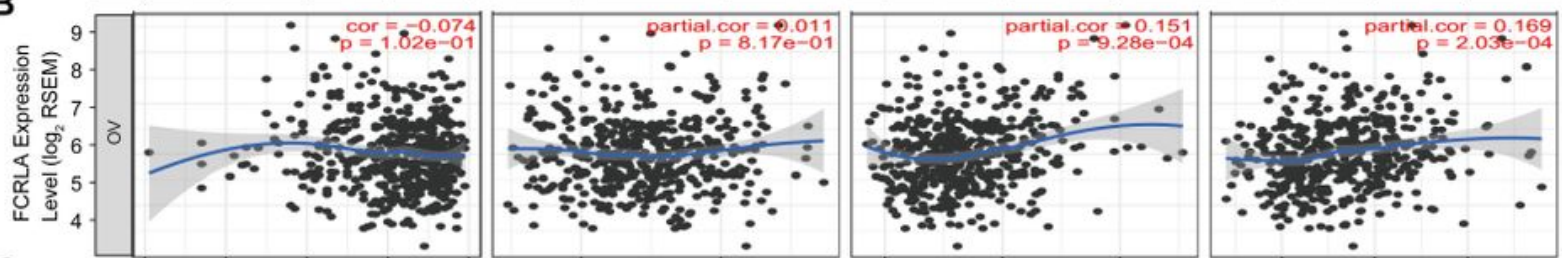

C
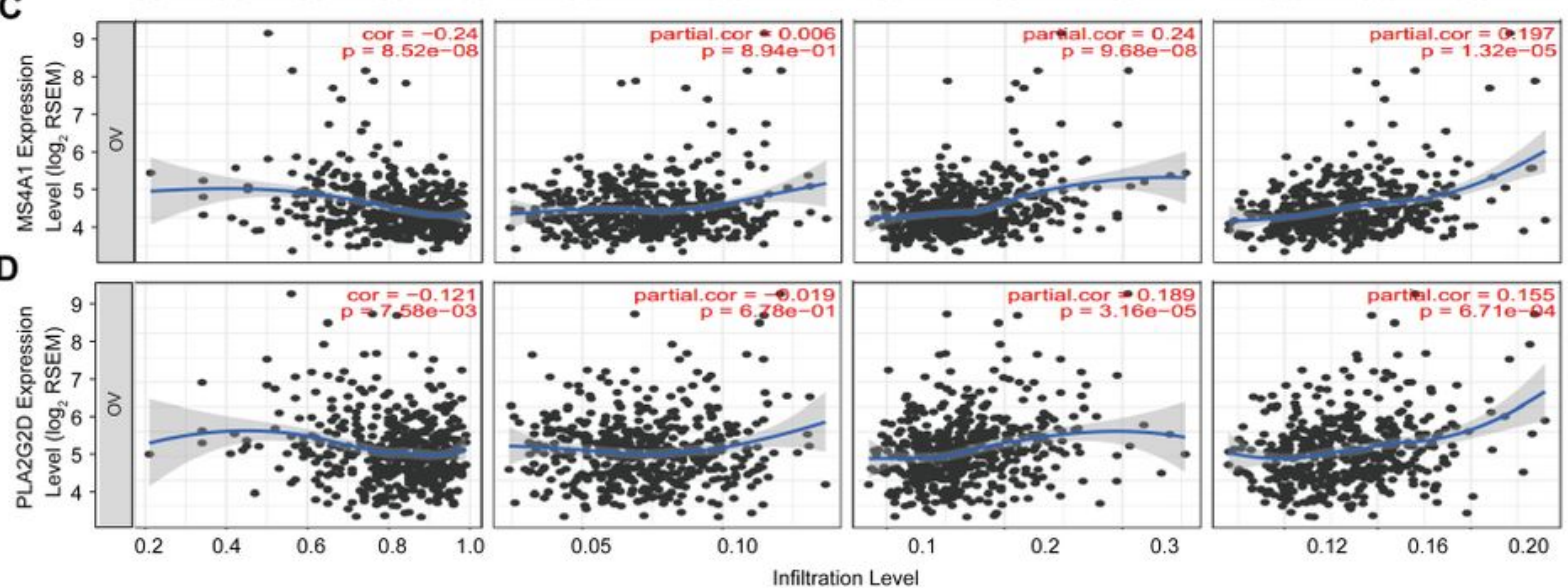

E

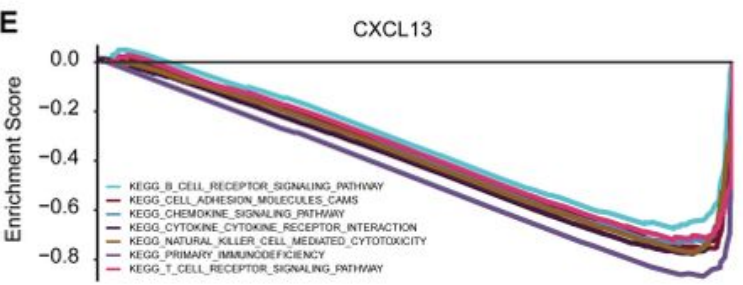

$\mathbf{F}$

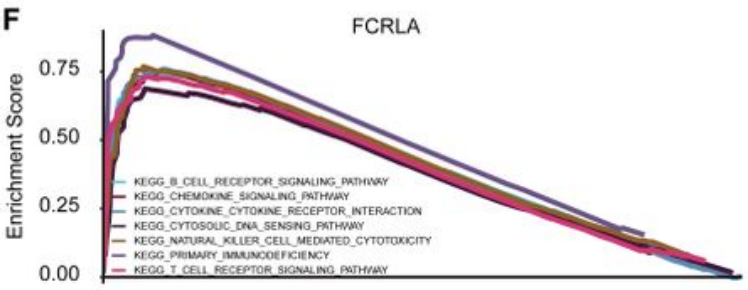

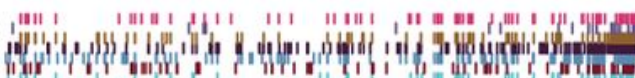
High expression<-----------> Low expression

G

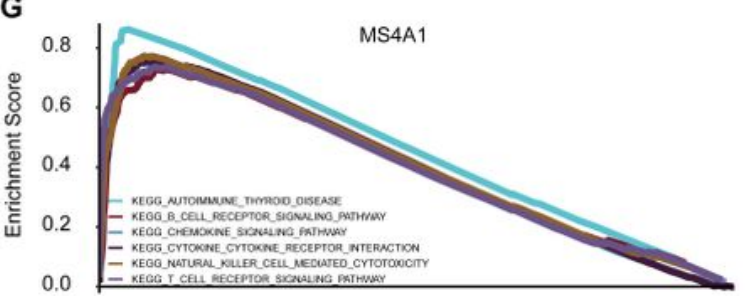

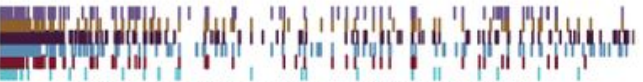
High expression<---------->>Low expression

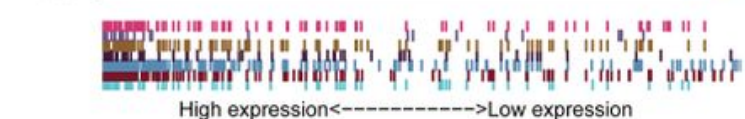

H

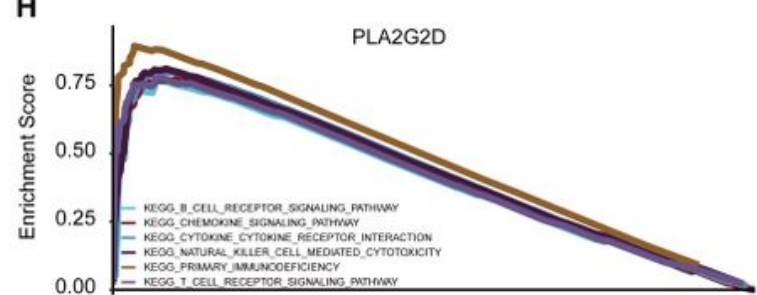

"

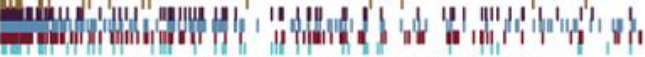
High expression<---------->>Low expression

Figure 6

CXCL13, FCRLA, MS4A1, and PLA2G2D correlated with immune status in OC. (A-D) The scatter plot shows the correlation between the expression of CXCL13, FCRLA, MS4A1, PLA2G2D, and the immune infiltration in OC samples from the TIMER website. (E-H) GSEA analyzed the correlation between the expression levels of four genes and the KEGG pathway. Genes expressed in the profile datasets were ranked by fold changes (high-expression/low-expression). GSEA correlation pathways were determined 
by the given algorithm. The vertical bars on the $x$-axis of the GSEA diagram represent the positions of genes in a given set in a sorted list. Positive and negative GSEA curves mean positive and negative enrichments, respectively.

A
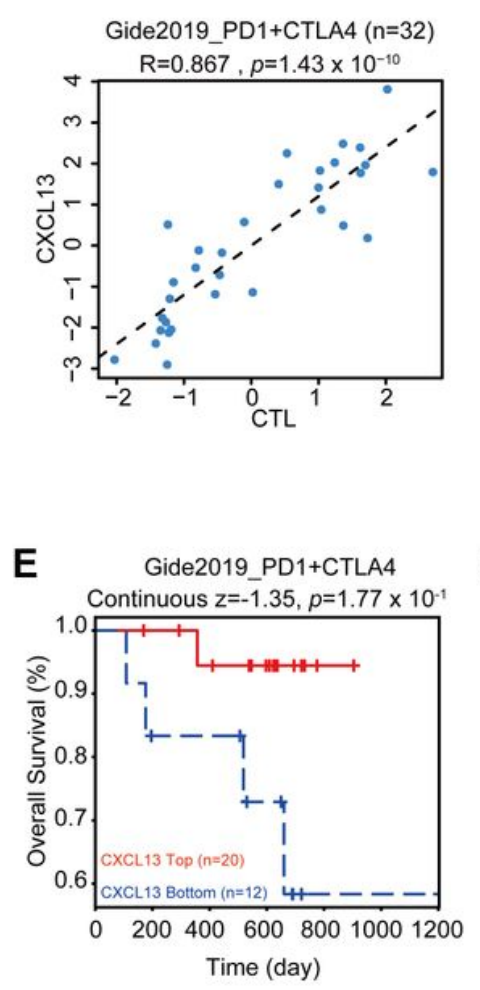

B

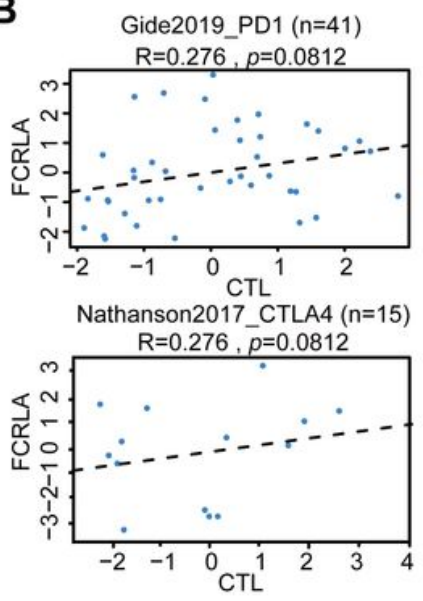

F Gide2019_PD1+CTLA4 $\quad$ G

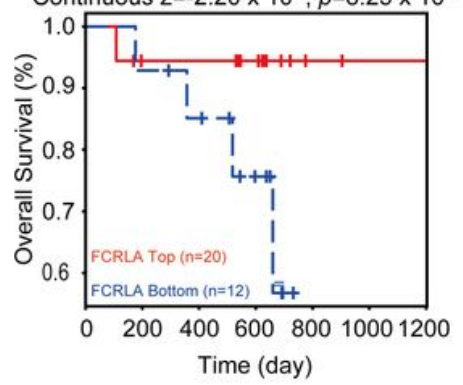

C
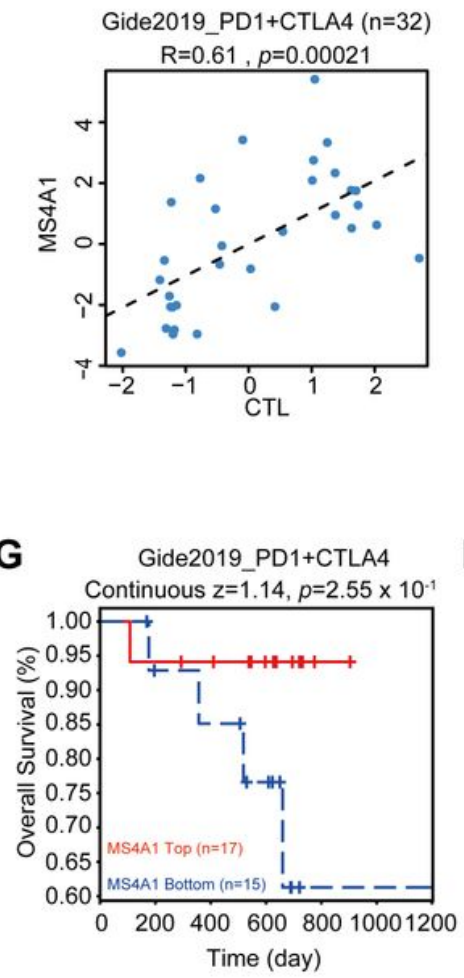

D

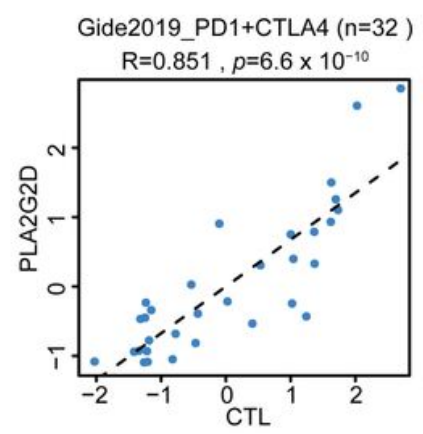

Figure 7

CXCL13, FCRLA, MS4A1, and PLA2G2D correlated with immunotherapy. (A-D) The scatter plot shows the correlation between the expression levels of CXCL13, FCRLA, MS4A1, PLA2G2D, and CTL infiltration. The $x$-axis shows the level of CTL infiltration in each melanoma sample. The Pearson correlation (R) between the plotted values is shown in the upper of each plot. $(E-H)$ The relation between the expression levels of CXCL13 (E), FCRLA (F), MS4A1 (G), and PLA2G2D (H) and the overall survival rate of melanoma patients treated with anti-PD1 and anti-CTLA4. The p-value was calculated by testing the association between high/low expression and overall survival with the two-sided Wald test in a Cox-PH regression. Samples were split according to these four gene expression levels, respectively. Each Kaplan-Meier plot presents tumors in two groups: 'High expression' (red) have above-average values among all samples, while 'Low expression' (blue) have values below average. The z-score of CXCL13, FCRLA, MS4A1, and PLA2G2D were calculated by dividing the interaction coefficient by its standard error. $\mathrm{P}<0.05$ in the cox- $\mathrm{PH}$ regression.

\section{Supplementary Files}

This is a list of supplementary files associated with this preprint. Click to download. 
- SupplementaryMaterial.pdf

- SupplementaryMaterial.pdf

- SupplementaryMaterial.pdf

- Tables1.xls

- Tables1.xls

- Tables1.xls

- Tables2.xls

- Tables2.xls

- Tables2.xls

- Tables3.xls

- Tables3.xls

- Tables3.xls 